\title{
Sputum Vitamin D Binding Protein (VDBP) GCIS/ IS Genotype Predicts Airway Obstruction: A Prospective Study in Smokers with COPD
}

This article was published in the following Dove Press journal: International Journal of Chronic Obstructive Pulmonary Disease

\begin{abstract}
Jing Gao ${ }^{1, *}$
Tanja Töröläl,*

Chuan-Xing Li (iD ${ }^{2}$

Steffen Ohlmeier (iD ${ }^{3}$

Tuula Toljamo ${ }^{4}$

Pentti Nieminen (iD) ${ }^{5}$

Noboru Hattori ${ }^{6}$

Ville Pulkkinen'

Hiroshi Iwamoto (iD ${ }^{6}$

Witold Mazur (iD)

'Heart and Lung Centre, Department of Pulmonary Medicine, University of Helsinki and Helsinki University Hospital, Helsinki, Finland; ${ }^{2}$ Pulmonomics Group, Respiratory Medicine Unit, Department of Medicine \& Centre for Molecular Medicine, Karolinska Institute, Stockholm, Sweden; ${ }^{3}$ Proteomics Core Facility, Biocentre Oulu, Faculty of Biochemistry and Molecular Medicine, University of Oulu, Oulu, Finland; ${ }^{4}$ Department of Pulmonary Medicine, Lapland Central Hospital, Rovaniemi, Finland; ${ }^{5}$ Medical Informatics and Statistics Group, University of Oulu, Oulu, Finland; ${ }^{6}$ Department of Molecular and Internal Medicine, Graduate School of Biomedical Sciences, Hiroshima University, Hiroshima, Japan
\end{abstract}

*These authors contributed equally to this work

Correspondence: Jing Gao

Heart and Lung Centre, Department of Pulmonary Medicine, University of Helsinki and Helsinki University Hospital, Finland

Tel +3584I707I559

Email jing.gao@helsinki.fi, Helsinki
Introduction: The vitamin D binding protein (VDBP, also known as GC-globulin) and vitamin $\mathrm{D}$ deficiency have been associated with chronic obstructive pulmonary disease (COPD). rs7041 and rs4588 are two single nucleotide polymorphisms of the VDBP gene, including three common allelic variants (GC1S, GC1F and GC2). Previous studies primarily assessed the serum levels of vitamin D and VDBP in COPD. However, less is known regarding the impact of the local release of VDBP on COPD lung function. Thus, we examined the association of sputum and plasma VDBP with lung function at baseline and at four years, and examined potential genetic polymorphism interactions.

Methods: The baseline levels of sputum VDBP, plasma VDBP and plasma 25-OH vitamin D, as well as the GC rs4588 and rs7041 genotypes, were assessed in a 4-year Finnish follow-up cohort $(n=233)$ of non-smokers, and smokers with and without COPD. The associations between the VDBP levels and the longitudinal decline of lung function were further analysed. Results: High frequencies of the haplotypes in rs7041/rs4588 were homozygous GC1S/1S (42.5\%). Higher sputum VDBP levels in stage I and stage II COPD were observed only in carriers with GC1S/1S genotype when compared with non-smokers $(\mathrm{p}=0.034$ and $\mathrm{p}=0.002$, respectively). Genotype multivariate regression analysis indicated that the baseline sputum VDBP and FEV1/FVC ratio at baseline independently predicted FEV1\% at follow-up.

Discussion and Conclusion: The baseline sputum VDBP expression was elevated in smokers with COPD among individuals with the GC1S/1S genotype, and predicted followup airway obstruction. Our results suggest that the GC polymorphism should be considered when exploring the potential of VDBP as a biomarker for COPD.

Keywords: vitamin D binding protein, VDBP, genotype, COPD, prospective study, sputum

\section{Plain Language Summary}

The vitamin D binding protein (VDBP, also known as GC-globulin) and vitamin D deficiency have been associated with chronic obstructive pulmonary disease (COPD). rs7041 and rs4588 are two single nucleotide polymorphisms of the VDBP gene, including three common allelic variants (GC1S, GC1F and GC2). Previous studies primarily assessed the serum levels of vitamin D and VDBP in COPD. However, less is known regarding the impact of the local release of VDBP on COPD lung function. Thus, we aimed to examine the association of sputum and plasma VDBP with lung function at baseline and at four years, and the interaction with genetic polymorphisms in a Finnish four-year follow-up cohort. Sputum VDBP expression in rs7041 was elevated in smokers with COPD and predicted with follow-up airway obstruction in individuals with the GC1S/1S genotype. This suggests that GC polymorphism should be considered when the potential of VDBP as a biomarker for COPD is further evaluated. 


\section{Introduction}

Chronic obstructive pulmonary disease (COPD) is one of the leading causes of morbidity and mortality worldwide, ${ }^{1,2}$ and genetic susceptibility also plays a role in airway disease. ${ }^{3}$ Although cigarette smoking is the main environmental risk factor for COPD, it is still unknown why airway obstruction is not present in all smokers. Exposure to sunlight and dietary intake are the main means by which to acquire vitamin $\mathrm{D}$ in humans. ${ }^{4}$ Acquired vitamin D results in 25-hydroxyl vitamin D (25-OH vitamin D), ${ }^{5,6}$ and approximately $85 \%$ of circulating $25-\mathrm{OH}$ vitamin $\mathrm{D}$ is tightly bound to the vitamin $\mathrm{D}$ binding protein (VDBP). ${ }^{4}$ VDBP, known as a group-specific component (GC) globulin, $\sim 58 \mathrm{kDa}$ in size, ${ }^{7}$ is best known as the main carrier of vitamin D metabolites, which could affect COPD through various overlapping mechanisms, ${ }^{8}$ VDBP carries immunomodulatory functions relevant in the lung and the majority of evidence relates to COPD. ${ }^{8}$ VDBP relates to macrophage activation and neutrophil chemotaxis, and enhances the chemotactic activity of the complement peptide C5a and its breakdown product C5a des-Arg. ${ }^{8-10}$ Furthermore, higher sputum VDBP levels correlated with an increased macrophage activation in patients with alpha-1-antitrypsin deficiency. ${ }^{11}$ Previous studies primarily assessed the serum levels of VDBP in COPD, whereas less is known regarding the impact of the local release of VDBP on COPD lung function. In the Evaluation of COPD Longitudinally to Identify Predictive Surrogate Endpoints (ECLIPSE) study, serum VDBP neither predicted the progression of COPD, such as changes in lung function and exacerbations, ${ }^{12}$ nor correlated with emphysema or forced expiratory volume in 1 $\mathrm{s}$ (FEV1). ${ }^{13}$ Yet, higher serum levels of VDBP were associated with a lower FEV1 in alpha-1-antitrypsin deficient patients in a cross-sectional study. ${ }^{11}$ Sputum VDBP levels were significantly elevated in vitamin $\mathrm{D}$ deficient patients with bronchiectasis and also associated with increased airway inflammation. ${ }^{14}$ In our previous proteomic screening study, we identified elevated VDBP in the sputum of smokers with COPD compared to non-smokers and smokers without COPD. ${ }^{15-17}$

Single nucleotide polymorphisms (SNPs) in the VDBP encoding genetic variations in the GC have been associated with $\mathrm{FEV}^{18}$ and levels of $25-\mathrm{OH}$ vitamin $\mathrm{D}^{19,20}$ in genome-wide association studies. In candidate gene approaches, two common SNPs in the GC gene, rs7041 [T->G, Asp (432) Glu] and rs4588[C->A, Thr (436) Lys], have been associated with a risk for $\mathrm{COPD}^{21}$ and $25-\mathrm{OH}$ vitamin D levels. $^{22-24}$ Combinations of these GC SNPs result in three common variants: GC1F, GC1S and GC2, each of which differ in their amino acid sequences at positions 432 and $436 .{ }^{8,25}$ The alleles included (a) GC1F (rs7041-T/rs4588-C); (b) GC1S (rs7041-G/rs4588-C); and (c) GC2 (rs7041-T/rs4588-A), inclu1F, 1S/1F, 1S/1S, 2/2) or heterozygous $(1 \mathrm{~F} / 2,1 \mathrm{~F} / 1 \mathrm{~S}, 1 \mathrm{~S} / 2)$ genotype. $^{26-28}$ In recent meta-analyses, the GC1S and GC1F variants associated with COPD in Asians, although results among Caucasians remain inconsistent. ${ }^{19,21,29}$ GC1F and GC1S are hypothesised to deliver $25-\mathrm{OH}$ vitamin $\mathrm{D}$ to target tissues more efficiently than GC2. ${ }^{30}$ Furthermore, GC2 is less able to activate macrophages ${ }^{31}$ resulting in reduced macrophage functioning in GC2 carriers when compared to carriers of GC1F and GC1S. This could result in reduced pulmonary inflammation in GC2 carriers and may in part explain results from recent meta-analyses showing that the GC2 variant protects against COPD in Asian populations. ${ }^{21,32}$ Thus, a comprehensive investigation of these SNPs' stratification in sputum and plasma VDBP expression in a longitudinal COPD study is warranted.

Taken together, vitamin D and VDBP are involved in a complex metabolic pathway that could affect COPD through different overlapping mechanisms. The GC gene variants may affect COPD by altering the $25-\mathrm{OH}$ vitamin $\mathrm{D}$ levels and by modulating the direct effects of VDBP on pulmonary inflammation. We propose that sputum VDBP might represent a more specific biomarker of local inflammation and lung damage in COPD than circulating VDBP. We hypothesised that the local release of VDBP independent of vitamin D levels influences longitudinal outcomes in COPD. Thus, our study aimed to examine the association of sputum and plasma VDBP with smoking and the trajectory of lung functioning over a four-year follow-up period in smokers with and without COPD, including the effects of a GC polymorphism.

\section{Methods}

\section{Participants and Samples}

Our cohort of 233 subjects (58.4\% men), which at baseline consisted of healthy non-smokers (NS, $n=32$ ), healthy smokers without COPD (HS, $\mathrm{n}=157$ ), smokers with COPD stage I (COPD st I, $\mathrm{n}=21$ ) and stage II and III (COPD st II,$+ \mathrm{n}=23$ ), forms a part of a longitudinal study conducted in Finland. ${ }^{33}$ All participants reported that they felt healthy, took no medications and were not affected by any other exposures or comorbidities. Upon enrolment, no samples or spirometry were collected. Two years following enrolment, we assessed the smoking 
status, and baseline spirometry, plasma and sputum samples were collected. The exclusion criteria consisted of the presence of lung disease or another disease; the regular use of a medication; risk factors for lung disease such as allergies, infections and exposures; a history of asthma or any previous lung infection including pneumonia or bronchiectasis; a malignancy; and a viral infection during the previous two months. ${ }^{34,35}$ Thus, none of the participants had comorbidities or took medications nor were affected by any other exposures at the time of enrolment. Spirometry was conducted at baseline and at follow-up in all subjects. All smokers in the study had a cigarette smoking history of $\geq 10$ years. A COPD diagnosis was assessed at baseline according to the 2007 GOLD criteria. The workflow appears in Figure 1.

Peripheral whole venous blood was collected in EDTA tubes and plasma was prepared by centrifugation and stored at $-80^{\circ} \mathrm{C}$ until analysis. Sputum samples were processed using the sputum plug method as described elsewhere. ${ }^{36,37}$ Only representative sputum samples containing less than $70 \%$ squamous epithelial cells were included for analysis.

\section{Proteomics, Western Blot and ELISA}

We described the detection and identification of VDBP $(\mathrm{n}=21)$ using two-dimensional difference gel electrophoresis
(2D-DIGE) and mass spectrometry (MS) in purified sputum (5 $\mu \mathrm{g} ; \mathrm{n}=21)$ in detail in our earlier study. ${ }^{15}$ Immunoblotting of the sputum samples $(n=21)$ relied on Western blot $(n=14)$ as previously described. ${ }^{38}$ VDBP levels and $25-\mathrm{OH}$ vitamin D levels $(n=233)$ were analysed using the Human Vitamin DBP Quantikine ELISA kit (R\&D Systems) according to the manufacturer's instructions and the $25-\mathrm{OH}$ vitamin D ELISA kit (Biovendor) according to the manufacturer's instructions. The limits of detection in VDBP and 25-OH vitamin D were $3.74 \mathrm{ng} / \mathrm{mL}$ and $1.9 \mathrm{ng} / \mathrm{mL}$, respectively.

\section{Genotyping of rs704I and rs4588}

We investigated the distribution of two common GC genotypes (rs4588 and rs7041) among the study groups as well as their effect on VDBP levels. DNA was extracted from whole blood using the FlexiGene DNA kit (Qiagen) according to the manufacturer's instructions. Genotyping of GC rs7041 and rs4588 was performed using Sanger sequencing. Amplification of the samples was performed in a $20 \mu \mathrm{L}$ volume containing $\sim 20 \mathrm{ng}$ of sample DNA, $0.25 \mu \mathrm{M}$ of each primer (VDBP_F primer TCATTGCAAAGACAGCCAAG and VDBP_R TCTCGAAGAGGCATGTTTCA) and the Phusion High-Fidelity PCR Master Mix (Thermo

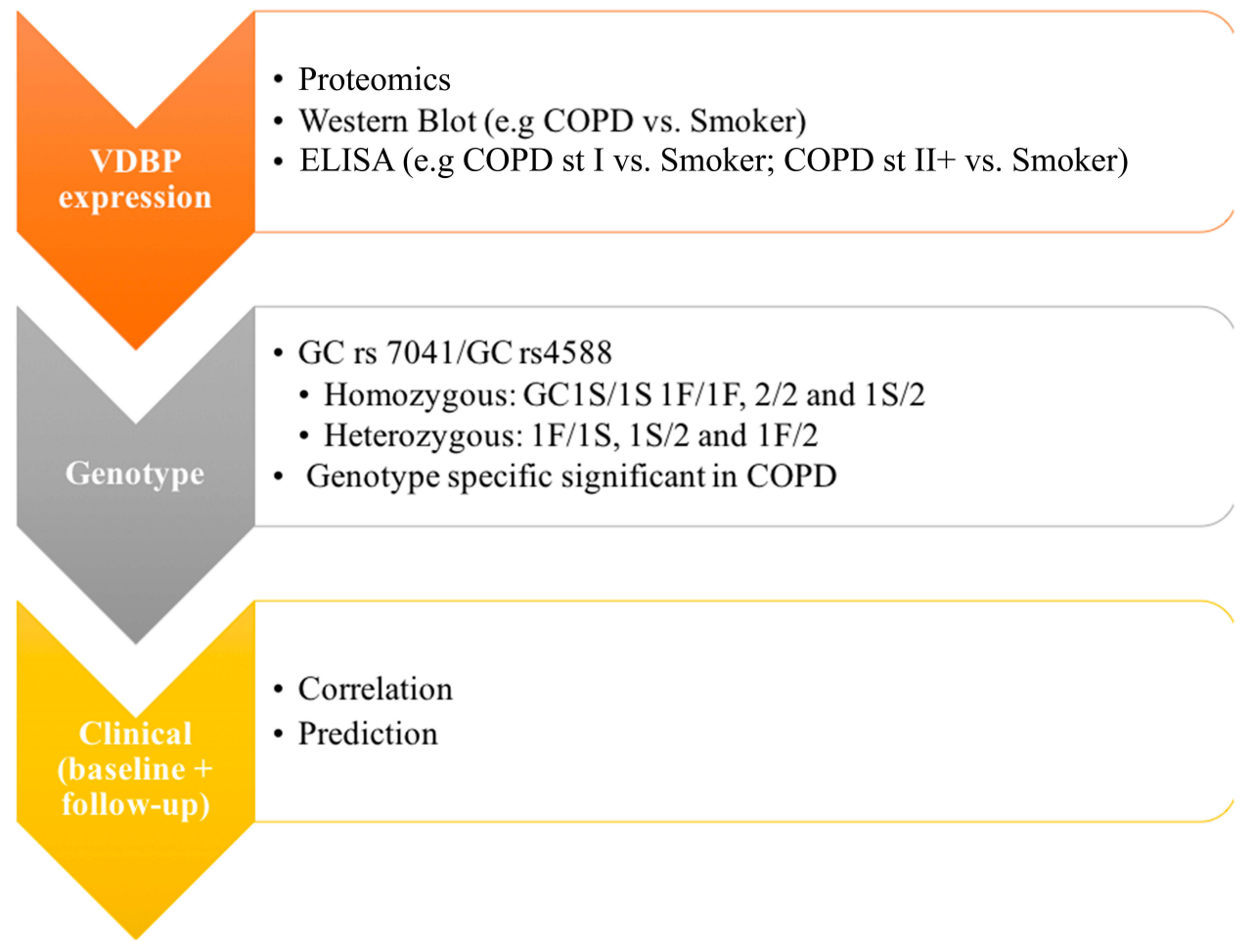

Figure I The workflow of the study design.

Abbreviations: VDBP, vitamin D binding protein; GC, VDBP also known as group-specific component-globulin; GCIF, rs704I-T/rs4588-C; GCIS, rs704I-G/rs4588-C; GC2, rs704I-T/rs4588-A; COPD, chronic obstructive pulmonary disease; Smokers, smokers without COPD. 
Scientific Inc). Initial denaturation took place at $98^{\circ} \mathrm{C}$ for $30 \mathrm{~s}$, followed by 30 cycles at $98^{\circ} \mathrm{C}$ for $10 \mathrm{~s}$, at $63^{\circ} \mathrm{C}$ for $30 \mathrm{~s}$ and at $72^{\circ} \mathrm{C}$ for $15 \mathrm{~s}$. The final extension took place at $72^{\circ} \mathrm{C}$ for $10 \mathrm{~min}$, followed by holding at $10^{\circ} \mathrm{C}$. DNA template purification was performed with resin and Performa DTR v3 filter plates (EdgeBio). The BigDye v.3.1 chemistry (Applied Biosystems) sequencing reaction was performed for purified DNA templates $(2 \mu \mathrm{L})$ with VDBP_F and VDBP_R primers at $96^{\circ} \mathrm{C}$ for $60 \mathrm{~s} ; 29$ cycles took place at $96^{\circ} \mathrm{C}$ for $10 \mathrm{~s}$, at $60^{\circ} \mathrm{C}$ for $10 \mathrm{~s}$, at $60^{\circ} \mathrm{C}$ for $4 \mathrm{~min}$ followed by holding at $10^{\circ} \mathrm{C}$ in a volume of $10 \mu \mathrm{L}$. The sequencing reaction purification was performed with Performa DTR v3 filter plates (EdgeBio) and electrophoresis was carried out using the ABI3730xl DNA Analyzer. Base calling was performed with the Sequencing Analysis software version 5.2 (Applied Biosystems), while the SNP analysis for rs7041 and rs4588 was performed using the variant reporter software version 1.0 (Applied Biosystems).

\section{Statistical Analysis}

The distribution of the basic subject characteristics was calculated using the means and standard deviations (SD) or percentages, as well as with the medians and interquartile ranges (IQRs). Comparisons between continuous variables among the study groups were calculated using the Kruskal-Wallis test, followed by the Mann-Whitney $U$-test, whereas the $\chi^{2}$ test was used for categorical variables. The Spearman's rank correlation was used to evaluate the association between biomarker concentrations with other variables. To obtain an independent predictor for follow-up FEV1\%, we performed multivariate regression analyses. All statistical analyses were performed using IBM's SPSS Statistics version 21 (SPSS, Chicago, IL, USA) and GraphPad Prism version 6.00 (GraphPad Software, San Diego, CA, USA). We considered $\mathrm{p}<0.05$ as statistically significant.

\section{Ethics Statement}

All the samples were analysed anonymously. This study was approved by the Ethics Committee of the Lapland Central Hospital (4 June 2003 and 31 October 2006), and all participants provided their written informed consent.

\section{Results}

\section{Participant Characteristics}

The baseline and follow-up characteristics of the participants in each of the study groups appear in Table 1 . The mean value of age was 53.9 years at baseline, and COPD stage II+ patients were significantly older than participants in the other groups. The predicted FEV1\% was significantly different between all groups at baseline and at follow-up. We found that $66.5 \%$ of participants at baseline were current smokers, and the $37.8 \%$ participants had a cough.

Table 2 summarises the frequencies of the GC rs 4588 and GC rs7041 genotypes in the different study groups. The occurrence of the rs 7041 and rs 4588 genotypes did not significantly differ between the COPD and non-COPD groups ( $p=0.216$ and $p=0.141$, respectively). The frequencies of the haplotypes in rs7041/rs4588 included homozygous $\mathrm{GC} 1 \mathrm{~S} / 1 \mathrm{~S}(42.5 \%), 1 \mathrm{~F} / 1 \mathrm{~F}(2.1 \%)$ and $2 / 2$ $(2.6 \%)$ as well as heterozygous $1 \mathrm{~F} / 1 \mathrm{~S}(15.9 \%), 1 \mathrm{~F} / 2$ $(36.5 \%)$ and $1 \mathrm{~S} / 2(0.4 \%)$.

\section{Secreted VDBP Isoform I Is Elevated in the Sputum of Smokers with COPD}

In our previous proteomic screening study, we observed higher sputum VDBP levels in smokers with COPD stage II compared to healthy non-smokers and smokers without COPD ${ }^{15}$ We detected changes in VDBP at two spots with similar expression profiles indicating similar regulatory mechanisms. Because different VDBP variants including three isoforms as well as full-length and secreted proteins have been described, we characterised the altered VDBP variant in more detail. The separation of VDBP in the 2D gel at pI 5.10/53 kDa (spot 1) and pI 5.14/53 kDa (spot 2) together with a sequence coverage of amino acids (31-363) obtained through MS for both spots indicated the presence of isoform 1 (Figure 2A). Since none of the detected peptides covered the N-terminal signal peptide (amino acids 1 16), this points to the presence of the secreted isoform 1 in the sputum (Figure 2B). This was further confirmed in immunoblotting experiments with sputum samples from healthy nonsmokers and smokers with COPD (Figure 2C).

\section{Sputum VDBP Levels Were Elevated in Patients with COPD}

We present the sputum VDBP levels as the medians with IQRs. The sputum VDBP levels were significantly elevated among smokers without COPD (129.4 [59.0 301.0] ng/mL), with COPD stage I (120.8 [92.3 305.7] ng/mL), and with COPD stage II $+(223.2[97.3 \sim 987.0] \mathrm{ng} / \mathrm{mL})$ when compared to the levels of VDBP in the sputum of healthy non-smokers (48.1 [25.7 150.4] ng/mL; Figure 3A). In addition, the sputum VDBP levels were elevated among smokers with COPD 
Table I Clinical Characteristics of Study Subjects at Baseline and at the Four-Year Follow-Up ( $=233)$

\begin{tabular}{|c|c|c|c|c|c|}
\hline & Non-Smokers & Smokers & COPD St I & COPD St II+ & \multirow[t]{2}{*}{ P-value } \\
\hline & $(n=32)$ & $(n=157)$ & $(n=21)$ & $(n=23)$ & \\
\hline \multicolumn{6}{|l|}{ Baseline } \\
\hline Subjects, $n$ & 32 & 157 & 21 & 23 & \\
\hline Gender (M/F) & $12 / 20$ & $86 / 7 \mid$ & $18 / 3$ & $20 / 3$ & $<0.00 \mathrm{I} \#$ \\
\hline Age years & $54.9(9.6)$ & $52.1(9.0)$ & $55.1(8.3)$ & $62.7(5.8)$ & $<0.00 I^{*}$ \\
\hline BMI & $26.2(3.6)$ & $26.9(3.8)$ & $26.2(3.3)$ & $28.3(4.4)$ & 0.259 \\
\hline Smoking status & & & & & $0.003^{*}$ \\
\hline Former smoker n (\%) & - & $33(21.0)$ & I (4.8) & $7(30.4)$ & \\
\hline Current smoker n (\%) & - & $124(79.0)$ & $19(90.5)$ & $12(52.2)$ & \\
\hline Pack-years & - & $28.4(14.1)$ & $35.5(12.7)$ & $43.1(13.0)$ & $<0.00 I^{*}$ \\
\hline Symptom & & & & & $<0.00 I^{*}$ \\
\hline Cough n (\%) & $4(12.5)$ & $61(38.9)$ & $9(42.9)$ & $14(60.9)$ & \\
\hline Sputum n (\%) & $9(28.1)$ & 91 (58.0) & $9(42.9)$ & $17(73.9)$ & \\
\hline \multicolumn{6}{|l|}{ Post-bronchodilator } \\
\hline FVC L & $3.8(0.8)$ & $4.0(1.0)$ & $4.8(0.8)$ & $3.6(0.7)$ & $<0.00 I^{*}$ \\
\hline Predicted FVC \% & $102.3(12.3)$ & $96.3(12.1)$ & $104.9(0.1)$ & $86.6(11.2)$ & $<0.00 I^{*}$ \\
\hline FEVI L & $3.2(0.6)$ & $3.3(0.8)$ & $3.2(0.5)$ & $2.1(0.5)$ & $<0.00 I^{*}$ \\
\hline Predicted FEVI\% & $106.5(14.6)$ & $96.5(12.5)$ & $87.3(5.2)$ & $63.8(I 1.4)$ & $<0.00 I^{*}$ \\
\hline FEVI/FVC \% & $84.5(5.3)$ & $81.6(5.4)$ & $67.2(2.7)$ & $59.8(7.8)$ & $<0.00 I^{*}$ \\
\hline \multicolumn{6}{|l|}{ At the 4-year follow-up } \\
\hline \multicolumn{6}{|l|}{ Post-bronchodilator } \\
\hline FVC L & $3.6(0.8)$ & $3.8(0.9)$ & $4.5(0.8)$ & $3.4(0.7)$ & $<0.00 I^{*}$ \\
\hline Predicted FVC \% & $100.4(13.4)$ & $96.2(11.8)$ & $101.5(9.3)$ & $85.6(14.9)$ & $<0.00 I^{*}$ \\
\hline FEVI L & $2.9(0.6)$ & $3.0(0.8)$ & $2.8(0.5)$ & $1.8(0.6)$ & $<0.00 I^{*}$ \\
\hline Predicted FEVI\% & $100.8(13.9)$ & $90.8(13.4)$ & $78.7(7.6)$ & $56.4(14.8)$ & $<0.00 I^{*}$ \\
\hline FEVI/FVC \% & $81.4(4.7)$ & $76.6(7.1)$ & $62.8(6.5)$ & $53.2(9.3)$ & $<0.00 I^{*}$ \\
\hline
\end{tabular}

Notes: The demographic characteristics are presented as the mean (standard deviation) unless otherwise stated. Differences were analysed using the Kruskal-Wallis $H$-test* and the chi-square test ${ }^{\#}$.

Abbreviations: BMI, body mass index; FEVI, forced expiratory volume in I s; FVC, forced vital capacity; COPD, chronic obstructive pulmonary disease; Smokers: smokers without COPD; COPD St I, smokers with COPD stage I; COPD St II+, smokers with COPD stage II+.

stage II+ in comparison to smokers without COPD $(\mathrm{p}=0.010)$ No significant difference emerged between study groups in the plasma VDBP and 25-OH vitamin D levels ( $\mathrm{p}>0.05$, respectively; Figure 3B and C). The 25-OH vitamin D levels were generally low in the plasma $(n=147)$. We found that 19 healthy non-smokers (59.3\%), 95 smokers without COPD (59.9\%), 16 patients with COPD stage I (76.2\%), and 17 patients with COPD stage II+ (73.9\%) had a vitamin D deficiency (plasma $25-\mathrm{OH}$ vitamin $\mathrm{D}<20 \mathrm{ng} / \mathrm{mL}$ ). The sputum VDBP levels did not significantly correlate with the plasma VDBP levels nor with the $25-\mathrm{OH}$ vitamin D levels in all subjects $(\mathrm{p}>0.05)$.

\section{Sputum VDBP Levels in COPD}

\section{Associated with GCIS/IS Genotype}

The sputum VDBP levels associated with the GC rs7041 and GC rs4588 genotypes in different study groups (Figure 4A), whereas no significant difference emerged between groups according to the genotypes in the plasma VDBP and 25-OH vitamin D (data not shown). Participants with the rs7041 GG genotype exhibited higher levels of sputum VDBP in COPD stage II+ $(355.5[110.2 \sim 973.5] \mathrm{ng} / \mathrm{mL})$ when compared to healthy non-smokers $(38.0[14.9 \sim 98.3] \mathrm{ng} / \mathrm{mL} ; \mathrm{p}=0.002$, Figure 4A). The rs4588 CC genotype showed higher levels of sputum VDBP in COPD stage II+ (242.9 [96.0 973.5] ng/ $\mathrm{mL})$ when compared to smokers (132.3 [72.8 268.2] ng/mL) and healthy non-smokers $(37.4$ [25.7 98.3] ng/mL; $\mathrm{p}=0.023$ and $p<0.001$, Figure 4B). The rs4588 CC genotype showed higher levels of sputum VDBP in COPD stage I (112.1 [98.7 123.2] $\mathrm{ng} / \mathrm{mL})$ than in healthy non-smokers $(\mathrm{p}=$ 0.003 , Figure 4B).

We also studied different genotype combinations of the rs7041/rs4588 genotype in homozygous (GC1S/1S, 1F/1F and 
Table 2 Frequencies of the GC rs704I and the GC rs4588 Genotypes in the Study Cohort

\begin{tabular}{|c|c|c|c|c|c|}
\hline & Non-Smokers & Smokers & COPD St I & COPD St II+ & Total \\
\hline & $(n=32)$ & $(n=157)$ & $(n=21)$ & $(n=23)$ & $(n=233)$ \\
\hline \multicolumn{6}{|l|}{ GC rs704I } \\
\hline $432^{\text {Asp/Asp }}(\mathrm{TT})$ & $5(15.6)$ & $15(9.6)$ & I (4.8) & $2(8.7)$ & $23(9.9)$ \\
\hline $432^{\text {Asp/Glu }}(\mathrm{TG})$ & $19(59.4)$ & $74(47.1)$ & $10(47.6)$ & $7(30.4)$ & $110(47.2)$ \\
\hline $432^{\text {Glu/Glu }}$ (GG) & $8(25.0)$ & $68(43.3)$ & $10(47.6)$ & $14(60.9)$ & $100(42.9)$ \\
\hline \multicolumn{6}{|l|}{ GC rs4588 } \\
\hline $436^{\text {Thr/Thr }}(C C)$ & $12(37.5)$ & $98(62.5)$ & $13(61.9)$ & $18(78.3)$ & $|4|(60.5)$ \\
\hline $436^{\text {Thr/Lys }}$ (CA) & $19(59.4)$ & $55(35.0)$ & $6(28.6)$ & $5(2 \mid .7)$ & $85(36.5)$ \\
\hline $436^{\text {Lys/Lys }}(\mathrm{AA})$ & I (3.1) & $4(2.5)$ & $2(9.5)$ & $0(0)$ & $7(3.0)$ \\
\hline \multicolumn{6}{|l|}{ GC rs704I/rs4577 } \\
\hline \multicolumn{6}{|l|}{ Homozygous } \\
\hline GCIS/GCIS (GG/CC) & $8(25.0)$ & $68(43.3)$ & $9(42.9)$ & $14(60.9)$ & $99(42.5)$ \\
\hline GCIF/GCIF (TT/CC) & I (3.1) & $3(1.9)$ & $0(0)$ & I (4.3) & $5(2.1)$ \\
\hline GC2/GC2 (TT/AA) & I (3.I) & $4(2.5)$ & I (4.8) & $0(0)$ & $6(2.6)$ \\
\hline \multicolumn{6}{|l|}{ Heterozygous } \\
\hline GCIF/GCIS (TG/CC) & $3(9.4)$ & 27 (I7.2) & $4(19.0)$ & $3(13.0)$ & 37 (I5.9) \\
\hline GCIF/GC2 (TG/CA) & $16(50.0)$ & $47(29.9)$ & $6(28.6)$ & $4(17.4)$ & $73(3 \mid .3)$ \\
\hline GCIF/GC2 (TT/CA) & $3(9.4)$ & $8(5.1)$ & $0(0)$ & I (4.3) & $12(5.2)$ \\
\hline GCIS/GC2 (GG/AA) & $0(0)$ & $0(0)$ & I (4.8) & $0(0)$ & I (0.4) \\
\hline
\end{tabular}

Notes: Data are presented as $\mathrm{n}(\%)$ unless otherwise stated.

Abbreviations: GC, VDBP also known as group-specific component-globulin; rs704I, T->G, Asp (432) Glu; rs4588, C->A, Thr (436) Lys; GCIF, rs704I-T/rs4588-C; GCIS, rs704I-G/rs4588-C; GC2, rs704I-T/rs4588-A; COPD, chronic obstructive pulmonary disease; Smokers, smokers without COPD; COPD St I, smokers with COPD stage I; COPD St II+, smokers with COPD stage II+.

2/2) and heterozygous $(1 \mathrm{~F} / 1 \mathrm{~S}, 1 \mathrm{~S} / 2$ and $1 \mathrm{~F} / 2)$ individuals. Among the studied genotypes in rs7041/rs4588, only GC1S/ $1 \mathrm{~S}$ showed different sputum VDBP levels between the groups with higher levels in COPD st I (112.1 [103.4 123.2] ng/mL) and COPD st II+ (355.5 [110.2 973.5.0] ng/mL) individuals compared to healthy non-smokers (38.0 [14.9 92.3] ng/mL; $\mathrm{p}=0.034$ and $\mathrm{p}=0.002$, respectively; Figure $4 C$ ).

\section{Sputum VDBP Levels Associated with Follow-Up FEVI\% Predicted in COPD in GCIS/IS Genotype}

Baseline sputum VDBP levels positively correlated with body mass index (BMI) and pack years among all participants, whereas we identified an inverse correlation between BMI and pack years associated with predicted FEV1\% at baseline and follow-up ( $p<0.05$, Table 3 ). In addition, the levels of plasma VDBP and vitamin D did not significantly correlate with lung function at baseline and follow-up in all subjects, nor rs7041 GG (1S), in rs4588 CC (1S) and GC 1S/1S (data not shown). Sputum VDBP expression with GC1S/1S negatively associated with the predicted FEV1\% at follow-up $(n=99$, $\mathrm{r}=-0.240, \mathrm{p}=0.017$; Figure 5) in the univariate analysis, but not in GC1F/1S $(\mathrm{n}=37, \mathrm{r}=-0.065, \mathrm{p}=0.703)$ and $\mathrm{GC} 1 \mathrm{~F} / 2$ $(\mathrm{n}=85, \mathrm{r}=-0.077, \mathrm{p}=0.485)$. Multivariate regression analysis indicated that baseline sputum VDBP and the FEV1/FVC ratio at follow-up independently predicted FEV1\% at follow-up in GC1S/1S genotype after adjusting for age, gender, BMI, smoking, plasma VDBP and vitamin D deficiency (Table 4). In contrast, those variables did not significantly predict FEV1\% at follow-up in GC1F/1S and GC 1S/2 (Table 4).

\section{Discussion}

Here, we found higher sputum VDBP levels in COPD patients associated with GC1S/1S compared to the levels among healthy non-smokers. Sputum VDBP emerged as an independent predictor for future airway obstruction in participants with the G1S/1S genotype. Plasma VDBP and $25-\mathrm{OH}$ vitamin $\mathrm{D}$ levels did not differ between the study groups, and did not significantly differ between COPD patients with and without vitamin $\mathrm{D}$ deficiency (plasma $25-\mathrm{OH}$ vitamin $\mathrm{D}<20 \mathrm{ng} / \mathrm{mL}$ ). In agreement with previous findings, ${ }^{11}$ the sputum VDBP levels did not correlate with the plasma VDBP levels. These observations suggest that the elevated sputum VDBP levels in COPD patients were related to the local release of VDBP independent of 


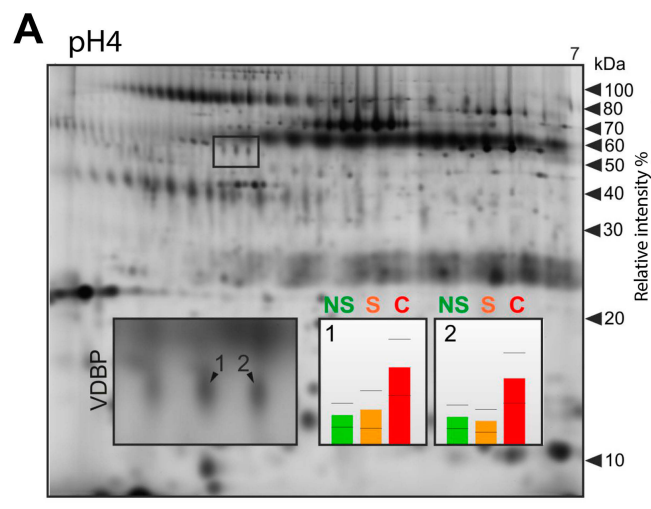

C

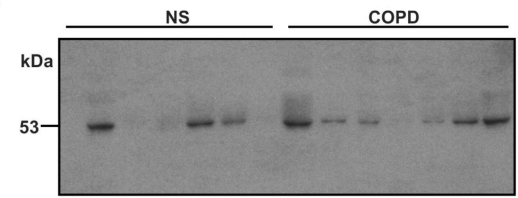

B

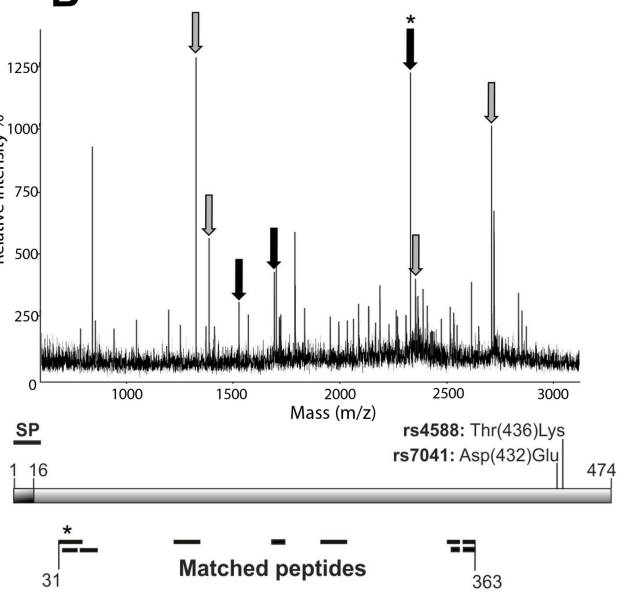

Figure 2 Secreted VDBP is upregulated in the sputum of smokers with COPD.

Notes: (A) Representative 2D gel of the sputum from a characteristic COPD patient (stage II). Altered protein spots of VDBP indicated by I and 2. Histogram corresponding to the spot numbers indicates the protein levels in non-smokers (NS), smokers (S) and COPD patients (C). (B) A representative mass spectrum (MS) of the altered VDBP. Peptides marked with grey arrows matched to all three isoforms whereas the black arrows matched only to isoforms $\mathrm{I}$ and 3 . The schematic presentation of VDBP shows the signal peptide (SP) and the sequence coverage of the spot-specific peptides detected by MS. The N-terminal peptide of the mature VDBP indicated by an asterisk. (C) Western blot of the sputum from non-smokers (NS, $n=7)$ and smokers with COPD stage II (COPD, $n=7)$.

Abbreviations: VDBP, vitamin D binding protein; COPD, chronic obstructive pulmonary disease; NS, non-smokers; S, smokers without COPD; C, COPD; MS, mass spectrum; SP, signal peptide.
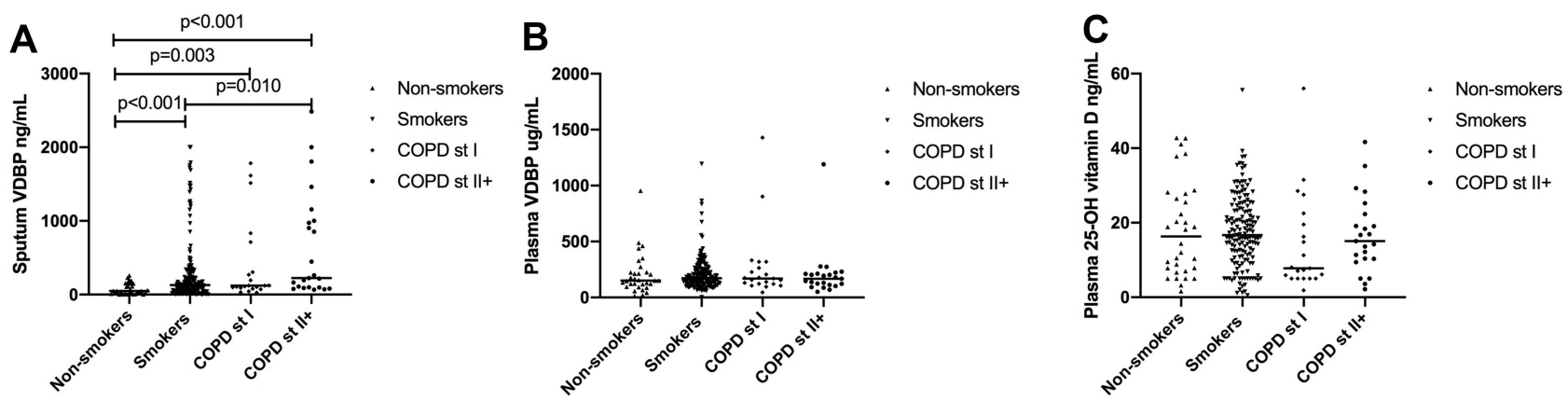

Figure 3 Levels of VDBP and 25-OH vitamin D at baseline for (A) sputum VDBP, (B) plasma VDBP and (C) plasma 25-OH vitamin D. Abbreviations: VDBP, vitamin D binding protein; COPD, chronic obstructive pulmonary disease; Smokers, smokers without COPD; COPD St I, smokers with COPD stage I; COPD St II+, smokers with COPD stage II+.

vitamin D carriage. In contrast, circulating VDBP may be affected by several confounding factors and may not represent a specific biomarker for COPD. These findings suggest that the sputum levels of VDBP might serve as a potential marker for COPD screening and predict COPD progression. In addition, genetic polymorphisms may affect the link between VDBP and the pathogenesis of COPD.

Previous studies identified significant associations between the sputum VDBP levels and markers of airway inflammation. ${ }^{14}$ Sputum VDBP may enhance macrophage activation $^{11}$ and neutrophil recruitment, ${ }^{9,10}$ resulting in immunomodulatory functions relevant to the lung. ${ }^{8}$ In our previous study, altered VDBP was detected in two spots with similar expression profiles indicating similar regulatory mechanisms. Here, the sputum VDBP levels were elevated in patients with COPD stage II+ compared with the levels in non-smokers and smokers overall and in the GC1S/1S genotype specifically. VDBP serves as the primary carrier of vitamin D metabolites, whereas sputum 


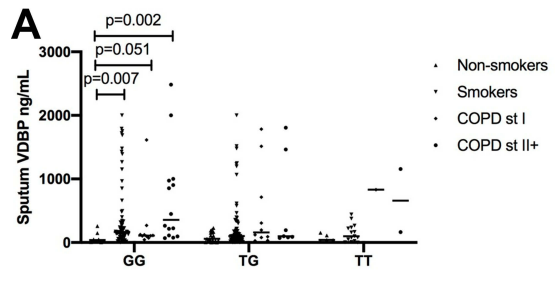

GC rs7041
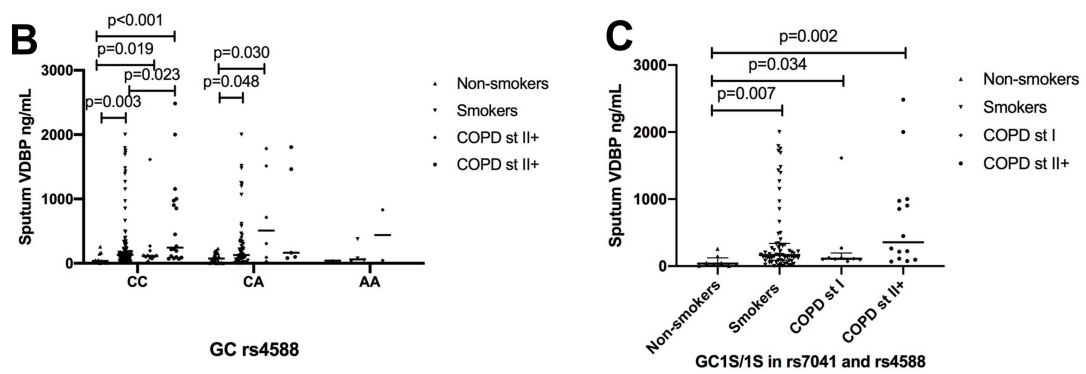

Figure 4 Sputum levels of VDBP according to the GC rs704I and rs4588 at baseline for (A) GC rs704I, (B) GC rs74588 and (C) GCIS/IS in rs704I/rs74588. Abbreviations: VDBP, vitamin D binding protein; GC, VDBP also known as group-specific component-globulin; rs704I, T->G, Asp (432) Glu; rs4588, C->A, Thr (436) Lys; GCIS, rs704I-G/rs4588-C; COPD, chronic obstructive pulmonary disease; Smokers, smokers without COPD; COPD St I, smokers with COPD stage I; COPD St II+, smokers with COPD stage II+.

VDBP did not correlate with plasma 25-OH vitamin D levels in our study. Notably, high sputum VDBP levels were associated with pack years at baseline, although smoking status did not emerge as an independent factor predicting sputum VDBP when compared with other variables. Thus, the elevated sputum VDBP levels in COPD patients may remain unaffected by the mere exposure to smoking, although the smoking-related disease process is affected. Furthermore, high sputum VDBP levels

Table 3 Correlation Between Sputum VDBP and Clinical Characteristics at Baseline and Follow-Up for Different Genotypes

\begin{tabular}{|l|l|l|l|}
\hline & $\begin{array}{l}\text { GC IS/IS } \\
(\mathbf{n = 9 9 )}\end{array}$ & $\begin{array}{l}\text { GC IF/IS } \\
\mathbf{( n = 3 7 )}\end{array}$ & $\begin{array}{l}\text { GC IF/2 } \\
\mathbf{( n = 8 5 )}\end{array}$ \\
\cline { 2 - 4 } & $\mathbf{r}$ & $\mathbf{r}$ & $\mathbf{r}$ \\
\hline Baseline & & & \\
Age & 0.121 & -0.229 & 0.05 I \\
BMI & 0.164 & 0.030 & $0.25 I^{*}$ \\
Gender & 0.077 & -0.127 & 0.181 \\
Pack years & 0.182 & -0.028 & $0.260^{*}$ \\
Predicted FVC \% & $-0.316^{* *}$ & -0.097 & 0.054 \\
Predicted FEVI\% & $-0.257^{*}$ & -0.042 & -0.052 \\
FEVI/FVC \% & 0.004 & -0.036 & -0.170 \\
Plasma VDBP & -0.023 & -0.061 & -0.109 \\
Plasma 25-OH vitamin & 0.035 & $-0.360^{*}$ & $-0.230^{*}$ \\
D & & & \\
Vitamin D deficiency & 0.011 & -0.304 & -0.200 \\
\hline Follow-up & & & \\
Predicted FVC \% & $-0.219^{*}$ & -0.005 & 0.068 \\
Predicted FEVI\% & $-0.240^{*}$ & -0.065 & -0.077 \\
FEVI/FVC \% & -0.064 & -0.158 & -0.200 \\
\hline
\end{tabular}

Notes: Data are analysed by Spearman correlation. ${ }^{*} p<0.05$ and ${ }^{* *} \mathrm{p}<0.001$. Abbreviations: VDBP, vitamin D binding protein; GC: VDBP also known as groupspecific component-globulin; GCIF, rs704I-T/rs4588-C; GCIS, rs704I-G/rs4588-C; GC2, rs704I-T/rs4588-A; BMI, body mass index; FEVI, forced expiratory volume in I s; FVC, forced vital capacity.

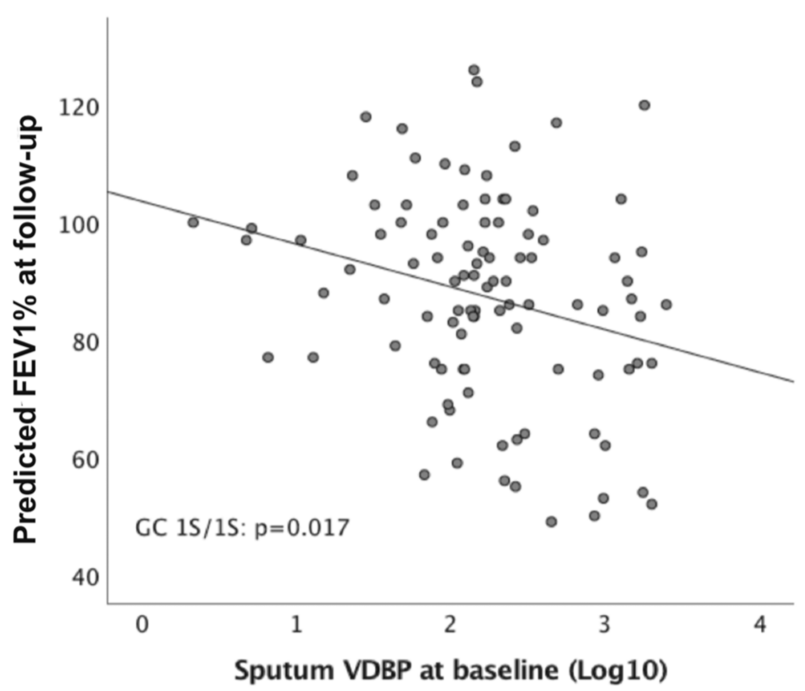

Figure 5 Correlation between sputum VDBP and predicted FEVI\% at follow-up based on GCIS/IS genotype.

Abbreviations: VDBP, vitamin D binding protein; GC, VDBP also known as groupspecific component-globulin; GCIS, rs704I-G/rs4588-C; FEVI: forced expiratory volume in I s; Log 10 , the logarithm base 10.

correlated with low predicted FEV1\% levels at baseline and at follow-up in patients with the $\mathrm{GC} 1 / 1 \mathrm{~S}$ genotype, but did not correlate with changes in lung function after four years at follow-up. This study predominantly included smokers without COPD and mild to moderate COPD. Persson et al showed that vitamin D deficiency correlates with the longitudinal lung function decline in moderate to very severe COPD. ${ }^{12}$ Yet, vitamin D deficiency has been shown to affect lung growth in early life. ${ }^{39}$ Our results identified an association between lung function and sputum VDBP, although further investigation is warranted in order to clarify whether altering lung VDBP associates with the development or progression of COPD.

In addition, previous results concerning the associations of circulating VDBP with COPD and lung function remain contradictory. ${ }^{11-13}$ Furthermore, the serum VDBP 
Table 4 Multivariate Regression Analysis of Predicted FEVI\% at Follow-Up as the Dependent Variable Among Subjects with Different Genotypes

\begin{tabular}{|l|l|l|l|l|l|}
\hline $\begin{array}{l}\text { Dependent FEVI\% } \\
\text { at Follow-Up }\end{array}$ & Beta & $\mathbf{t}$ & $\mathbf{p}$-value & $\mathbf{9 5 \%} \mathbf{C l}$ & \\
\cline { 4 - 6 } & & & & Lower & Upper \\
\hline $\begin{array}{l}\text { GCIS/IS ( }=99) \\
\text { Baseline FEVI/FVC } \\
\text { Baseline sputum VDBP }\end{array}$ & $\begin{array}{l}1.135 \\
-0.006\end{array}$ & $\begin{array}{l}7.878 \\
-2.429\end{array}$ & $\begin{array}{l}<0.001 \\
0.017\end{array}$ & $\begin{array}{l}0.849 \\
-0.011\end{array}$ & $\begin{array}{l}\text { I.42I } \\
-0.001\end{array}$ \\
\hline $\begin{array}{l}\text { GCIF/2 ( }=85) \\
\text { Baseline FEVI/FVC }\end{array}$ & 1.174 & 7.749 & $<0.001$ & 0.872 & 1.475 \\
Baseline pack-years & -0.235 & -2.96 & 0.004 & -0.392 & -0.077 \\
\hline $\begin{array}{l}\text { GCIF/IS ( } n=37) \\
\text { Baseline FEVI/FVC }\end{array}$ & 1.441 & 5.166 & $<0.001$ & 0.874 & 2.008 \\
BMI & -1.798 & -3.352 & 0.002 & -2.889 & -0.708 \\
\hline
\end{tabular}

Notes: Multivariate analysis was adjusted for age, gender, BMI, smoking, plasma VDBP and vitamin $D$ deficiency, respectively.

Abbreviations: VDBP, vitamin $D$ binding protein; GC: VDBP also known as groupspecific component-globulin; GCIF, rs704I-T/rs4588-C; GCIS, rs704I-G/rs4588C; GC2, rs704I-T/rs4588-A; BMI, body mass index; FEVI, forced expiratory volume in I s; FVC, forced vital capacity; Cl: confidence interval.

and vitamin D levels were inversely correlated, ${ }^{12-14}$ whereas another study found no correlation. ${ }^{11}$ The rs7041 SNP gene explained $71 \%$ to $75 \%$ of the measured variation in plasma $\mathrm{VDBP},{ }^{40}$ whereas we found no significant difference in the plasma VDBP rs7041 GG (1S) genotype among the groups in our study. Thus, circulating VDBP may be affected by several confounding factors and may not represent a specific biomarker for COPD.

The strength of this study lies in our systematic investigation of VDBP gene polymorphism in a well-characterised follow-up cohort using several types of samples. The frequencies of the different rs4588 and rs7041 genotypes agreed well with those previously detected in a Finnish population stored in the Sequencing Initiative Suomi database (http:// www.sisuproject.fi/). Our study also carries several limitations. First, the VDBP immunoassay may have been affected by polymorphisms with a lower VDBP detection in the GC1F variant. ${ }^{41}$ However, GC1F was rare in our cohort, in which only five individuals were homozygotes for this genotype. In addition, we must note that this study is insufficiently powered to allow for an examination of the genetic associations of GC variants with COPD. Second, the number of participants in the COPD groups was relatively small. A larger number of COPD samples and a validation cohort would be necessary to further confirm the association between the sputum VDBP and follow-up airway obstruction. Third, our sample was gender imbalanced, given that the COPD group included only six women participants.
Thus, we did not use gender further in our correlation analysis. Fourth, we found no significant correlation between the sputum VDBP and the longitudinal decline of lung function in a univariate analysis. We attribute this result to the small change in the absolute lung function value. Therefore, further investigation with follow-up period longer than 4 years in patients with various levels of severity would be needed to clarify this point.

In conclusion, our results show that the sputum VDBP expression levels are elevated in smokers with COPD and predict with follow-up airway obstruction in individuals with the GC1S/1S genotype. Gene polymorphism should be considered when the potential of VDBP as a biomarker for COPD is further evaluated.

\section{Acknowledgments}

This work was financially supported by EVO funding from the Helsinki University Central Hospital, the Sigrid Jusélius Foundation, the Finnish Anti-Tuberculosis Association Foundation, the Jalmari and Rauha Ahokas Foundation, and the Finnish Cultural Foundation, the Research Foundation of the Pulmonary Diseases, the Ida Montin Foundation, and the Väinö and Laina Kivi Foundation. We are thankful to the language centre of University of Helsinki providing the linguistic revision for this work. We wish to thank Vanessa L Fuller PhD, for linguistic revision of this work.

\section{Disclosure}

The authors report no conflicts of interest in this work.

\section{References}

1. Buist AS, McBurnie MA, Vollmer WM, et al. International variation in the prevalence of COPD (the BOLD Study): a population-based prevalence study. Lancet. 2007;370(9589):741-750. doi:10.1016/S01406736(07)61377-4

2. Lopez AD, Mathers CD, Ezzati M, Jamison DT, Murray CJ. Global and regional burden of disease and risk factors, 2001: systematic analysis of population health data. Lancet. 2006;367 (9524):1747-1757. doi:10.1016/S0140-6736(06)68770-9

3. Patel BD, Coxson HO, Pillai SG, et al. Airway wall thickening and emphysema show independent familial aggregation in chronic obstructive pulmonary disease. Am J Resp Crit Care. 2008;178(5):500-505. doi:10.1164/rcem.200801-059OC

4. Cho MC, Kim JH, Jung MH, et al. Analysis of vitamin D-binding protein (VDBP) gene polymorphisms in Korean women with and without endometriosis. Clin Exp Reprod Med. 2019;46(3):132-139. doi:10.5653/cerm.2019.00122

5. DeLuca HF. Overview of general physiologic features and functions of vitamin D. Am J Clin Nutr. 2004;80(6 Suppl):1689S-1696S. doi:10.1093/ajen/80.6.1689S

6. Holick MF. Vitamin D deficiency. $N$ Engl J Med. 2007;357 (3):266-281. doi:10.1056/NEJMra070553 
7. Song W, Wang X, Tian Y, Zhang X, Lu R, Meng H. GC gene polymorphisms and Vitamin D-binding protein levels are related to the risk of generalized aggressive periodontitis. Int $J$ Endocrinol. 2016;2016:5141089. doi:10.1155/2016/5141089

8. Chishimba L, Thickett DR, Stockley RA, Wood AM. The vitamin $\mathrm{D}$ axis in the lung: a key role for vitamin D-binding protein. Thorax. 2010;65(5):456-462. doi:10.1136/thx.2009.128793

9. Perez HD, Kelly E, Chenoweth D, Elfman F. Identification of the C5a des Arg cochemotaxin. Homology with vitamin D-binding protein (group-specific component globulin). J Clin Invest. 1988;82 (1):360-363. doi:10.1172/JCI113595

10. Kew RR, Webster RO. Gc-globulin (vitamin D-binding protein) enhances the neutrophil chemotactic activity of C5a and C5a des Arg. J Clin Invest. 1988;82(1):364-369. doi:10.1172/JCI113596

11. Wood AM, Bassford C, Webster D, et al. Vitamin D-binding protein contributes to COPD by activation of alveolar macrophages. Thorax. 2011;66(3):205-210. doi:10.1136/thx.2010.140921

12. Persson LJ, Aanerud M, Hiemstra PS, et al. Vitamin D, vitamin $\mathrm{D}$ binding protein, and longitudinal outcomes in COPD. PLoS One. 2015;10(3):e0121622. doi:10.1371/journal.pone.0121622

13. Berg I, Hanson C, Sayles H, et al. Vitamin D, vitamin D binding protein, lung function and structure in COPD. Respir Med. 2013;107 (10):1578-1588. doi:10.1016/j.rmed.2013.05.010

14. Chalmers JD, McHugh BJ, Docherty C, Govan JRW, Hill AT. Vitamin-D deficiency is associated with chronic bacterial colonisation and disease severity in bronchiectasis. Thorax. 2013;68(1):39U161. doi:10.1136/thoraxjnl-2012-202125

15. Ohlmeier S, Mazur W, Linja-Aho A, et al. Sputum proteomics identifies elevated PIGR levels in smokers and mild-to-moderate COPD. J Proteome Res. 2012;11(2):599-608. doi:10.1021/ pr2006395

16. Ohlmeier S, Mazur W, Salmenkivi K, Myllarniemi M, Bergmann U, Kinnula VL. Proteomic studies on receptor for advanced glycation end product variants in idiopathic pulmonary fibrosis and chronic obstructive pulmonary disease. Proteom Clin Appl. 2010;4 (1):97-105. doi:10.1002/prca.200900128

17. Ohlmeier S, Nieminen P, Gao J, et al. Lung tissue proteomics identifies elevated transglutaminase 2 levels in stable chronic obstructive pulmonary disease. Am J Physiol-Lung C. 2016;310(11):L1155L1165. doi:10.1152/ajplung.00021.2016

18. Wilk JB, Walter RE, Laramie JM, Gottlieb DJ, O'Connor GT. Framingham Heart Study genome-wide association: results for pulmonary function measures. BMC Med Genet. 2007;8(Suppl 1):S8. doi:10.1186/1471-2350-8-S1-S8

19. Wang TJ, Zhang F, Richards JB, et al. Common genetic determinants of vitamin D insufficiency: a genome-wide association study. Lancet. 2010;376(9736):180-188. doi:10.1016/S0140-6736(10)60588-0

20. Ahn J, Yu K, Stolzenberg-Solomon R, et al. Genome-wide association study of circulating vitamin D levels. Hum Mol Genet. 2010;19 (13):2739-2745. doi:10.1093/hmg/ddq155

21. Chen H, Zhang L, He Z, et al. Vitamin D binding protein gene polymorphisms and chronic obstructive pulmonary disease: a meta-analysis. J Thorac Dis. 2015;7(8):1423-1440. doi:10.3978/j. issn.2072-1439.2015.08.16

22. Janssens W, Bouillon R, Claes B, et al. Vitamin D deficiency is highly prevalent in COPD and correlates with variants in the vitamin D-binding gene. Thorax. 2010;65(3):215-220. doi:10.1136/ thx.2009.120659

23. Powe CE, Karumanchi SA, Thadhani R. Vitamin D-binding protein and vitamin D in blacks and whites. $N$ Engl J Med. 2014;370 (9):880-881. doi:10.1056/NEJMc1315850

24. Wilson RT, Bortner JD Jr., Roff A, et al. Genetic and environmental influences on plasma vitamin D binding protein concentrations. Transl Res. 2015;165(6):667-676. doi:10.1016/j.trsl.2014.08.003
25. Khanna R, Nandy D, Senapati S. Systematic review and meta-analysis to establish the association of common genetic variations in Vitamin D binding protein with chronic obstructive pulmonary disease. Front Genet. 2019;10:413. doi:10.3389/ fgene.2019.00413

26. Braun A, Bichlmaier R, Cleve H. Molecular analysis of the gene for the human vitamin-D-binding protein (group-specific component): allelic differences of the common genetic GC types. Hum Genet. 1992;89(4):401-406. doi:10.1007/BF00194311

27. Newton DA, Baatz JE, Kindy MS, et al. Insights image for vitamin D binding protein polymorphisms significantly impact vitamin D status in children. Pediatr Res. 2019;86(5):674. doi:10.1038/ s41390-019-0476-7

28. Newton DA, Baatz JE, Kindy MS, et al. Vitamin D binding protein polymorphisms significantly impact vitamin D status in children. Pediatr Res. 2019;86(5):662-669. doi:10.1038/s41390-019-0322-y

29. Wang YL, Kong H, Xie WP, Wang H. Association of vitamin D-binding protein variants with chronic obstructive pulmonary disease: a meta-analysis. Genet Mol Res. 2015;14(3):10774-10785. doi:10.4238/2015.September.9.16

30. Arnaud J, Constans J. Affinity differences for vitamin D metabolites associated with the genetic isoforms of the human serum carrier protein (DBP). Hum Genet. 1993;92(2):183-188. doi:10.1007/ BF00219689

31. Yamamoto N, Homma S. Vitamin D3 binding protein (group-specific component) is a precursor for the macrophage-activating signal factor from lysophosphatidylcholine-treated lymphocytes. Proc Natl Acad Sci U S A. 1991;88(19):8539-8543. doi:10.1073/pnas.88.19.8539

32. Xie X, Zhang Y, Ke R, et al. Vitamin D-binding protein gene polymorphisms and chronic obstructive pulmonary disease susceptibility: a meta-analysis. Biomed Rep. 2015;3(2):183-188. doi:10.3892/ br.2014.392

33. Toljamo T, Kaukonen M, Nieminen P, Kinnula VL. Early detection of COPD combined with individualized counselling for smoking cessation: a two-year prospective study. Scand J Prim Health Care. 2010;28(1):41-46. doi:10.3109/02813431003630105

34. Toljamo T, Hamari A, Sotkasiira M, Nieminen P. Clinical characteristics of COPD syndrome: a 6-year follow-up study of adult smokers. Ann Med. 2015;47(5):399-405. doi:10.3109/07853890.2015.1045551

35. Iwamoto H, Gao J, Pulkkinen V, Toljamo T, Nieminen P, Mazur W. Soluble receptor for advanced glycation end-products and progression of airway disease. BMC Pulm Med. 2014;14:68. doi:10.1186/ 1471-2466-14-68

36. Paggiaro PL, Chanez P, Holz O, et al. Sputum induction. Eur Respir J Suppl. 2002;37:3s-8s. doi:10.1183/09031936.02.00000302

37. Rytila P, Rehn T, Ilumets $H$, et al. Increased oxidative stress in asymptomatic current chronic smokers and GOLD stage 0 COPD. Respir Res. 2006;7:69. doi:10.1186/1465-9921-7-69

38. Gao J, Ohlmeier S, Nieminen P, et al. Elevated sputum BPIFB1 levels in smokers with chronic obstructive pulmonary disease: a longitudinal study. Am J Physiol Lung Cell Mol Physiol. 2015;309(1):L17-26. doi:10.1152/ajplung.00082.2015

39. Saadoon A, Ambalavanan N, Zinn K, et al. Effect of prenatal versus postnatal Vitamin D deficiency on pulmonary structure and function in mice. Am J Respir Cell Mol Biol. 2017;56(3):383-392. doi: $10.1165 / \mathrm{rcmb} .2014-0482 \mathrm{OC}$

40. Sun W, Kechris K, Jacobson S, et al. Common genetic polymorphisms influence blood biomarker measurements in COPD. PLoS Genet. 2016;12(8):e1006011. doi:10.1371/journal.pgen.1006011

41. Hoofnagle AN, Eckfeldt JH, Lutsey PL. Vitamin D-binding protein concentrations quantified by mass spectrometry. New Engl J Med. 2015;373(15):1480-1482. doi:10.1056/NEJMc1502602 


\section{Publish your work in this journal}

The International Journal of COPD is an international, peer-reviewed journal of therapeutics and pharmacology focusing on concise rapid reporting of clinical studies and reviews in COPD. Special focus is given to the pathophysiological processes underlying the disease, intervention programs, patient focused education, and self management protocols. This journal is indexed on PubMed Central, MedLine and CAS. The manuscript management system is completely online and includes a very quick and fair peer-review system, which is all easy to use. Visit http://www.dovepress.com/testimonials.php to read real quotes from published authors. 